\title{
Adaptación psicométrica del inventario de Texas revisado de duelo (ITRD) en población militar española joven adulta
}

\author{
Samper Lucena .E. ${ }^{1}$
}

Sanid. mil. 2011; 67 (3): 291-298; ISSN: 1887-8571

\begin{abstract}
RESUMEN
Introducción: Las pérdidas que acompañan la condición humana suponen un proceso muy difícil de incorporar en la vida, según la literatura científica uno de los acontecimientos vitales más estresantes. En la actualidad se ha incrementado el interés por estos temas y su demanda social. En las Fuerzas Armadas la actuación diaria de los hombres y mujeres que la integran, especialmente durante su participación en las misiones en el exterior, es la razón de su prestigio entre nuestros conciudadanos, pero también se sufren pérdidas de personas y heridos allí donde prestan servicio, lo que afecta a compañeros, familiares y amigos. Objetivos: En este trabajo se trata de estudiar psicométricamente el inventario de Texas revisado de duelo (ITRD) de Faschingbauer y cols., en personal militar joven adulto, revisando algunas de las variables que se han asociado en la literatura científica con el proceso psicológico estudiado. Aportar información relevante de apoyo en la consulta especializada o en situaciones de emergencia o catástrofe para la ayuda a personas que han perdido a seres queridos. Metodología: Se ha confeccionado un cuestionario para recoger los datos. Se aplicó a una muestra de adultos jóvenes, miembros de las Fuerzas Armadas Españolas, realizando el estudio psicométrico (análisis descriptivo, baremación mediante percentiles, y estudio factorial). Discusión: Se compararon los resultados obtenidos con los de otros estudios de autores tanto de la prueba original como de la versión utilizada en otras poblaciones de diferente origen. Conclusiones: La prueba utilizada es psicométricamente adecuada en población joven adulta de forma similar a lo encontrado en otros estudios con distinta población. Existen diferencias entre hombres y mujeres en el proceso de duelo. Que la clasificación de los baremos de la prueba se debe de adaptar a la población donde se aplica, clínica o no, diferentes grupos de edad, o diferentes orígenes culturales, ya que el proceso de duelo es distinto. El perfil de la persona con necesidades de apoyo especializado, en este estudio, es de una mujer sin tratamientos en salud mental y que ha perdido a un ser querido por una enfermedad. Se aporta un trabajo de interés para enriquecer los instrumentos diagnósticos psicológicos, en una población específica de las Fuerzas Armadas Españolas.
\end{abstract}

PALABRAS CLAVE: Duelo, Pérdida, ITRD, psicometría, atención psicológica, Fuerzas Armadas Españolas.

\section{Psychometric Adaptation of Texas Revised Inventory of Grief (TRIG) in a Spanish young adult military population SUMMARY}

Introduction: The losses that accompany the human condition are a difficult process to come to terms with, and according to scientific literature one of the most stressful life events. Today there is an increased interest in these issues and their social demand. The daily performance of male and female service members, especially during their participation in operations abroad, is the reason for the Armed Forces prestige among our fellow citizens. But operations also originate casualties and this affects colleagues, families and friends. Objectives: This paper seeks to explore psychometrically the Texas Revised Inventory of Grief (TRIG) by Faschingbauer et al., in young adult military personnel, reviewing some of the variables that have been associated in the literature with the psychological process studied. This paper provides relevant information to support the specialist consultation or in disaster situations to help people who have lost loved ones. Methodology: We prepared a questionnaire to collect data. It was applied to a sample of young adults, members of the Spanish Armed Forces, performing the psychometric study (descriptive analysis, scales by percentiles, and factorial study). Discussion: We compared the results with those of other studies by the authors of both the original test and the version used in other populations of different origin. Conclusions: The test used is psychometrically adequate for a young adult population as it has been proven in other studies with different populations. There are differences between men and women in the grieving process. The classification of the scales of the test must be adapted to the population where it is applied, clinical symptoms or not, different age groups, or different cultural backgrounds, since the grieving process is different in each group. The profile of the person in need of specialized support in this study is a woman with no mental health problems and who has lost a loved one to disease. This paper contributes to enrich the psychological diagnostic tools in a specific Spanish military population.

KEY WORDS: Grief, Loss, TRIG, Psychometrics, Psychological care, Spanish Armed Forces.

${ }^{1}$ Cap Psicólogo. Unidad Militar de Emergencias. Cuartel General (Base Aérea Torrejón de Ardoz). Madrid. España.

Dirección para correspondencia: Eduardo Samper Lucena. Unidad Militar de Emergencias. Cuartel General. Gabinete de Psicología. Base Aérea de Torrejón de Ardoz. 28850 Torrejón de Ardoz (Madrid).Tfno. 917487119. esamluc@ea.mde.es

Recibido: 17 de mayo de 2010

Aceptado: 17 de febrero de 2011

\section{INTRODUCCIÓN}

En la vida se sufren pérdidas continuamente, desde la niñez se está perdiendo objetos, capacidades y funciones importantes para las personas, hasta llegar a la pérdida de seres queridos. La muerte de un ser querido es uno de los acontecimientos más traumáticos y estresantes para las personas ${ }^{1}$. 
En la actualidad, el proceso de envejecimiento de la población y su trascendencia en la planificación de los sistemas sanitarios y de seguridad social, así como el creciente interés en la medición de las condiciones de vida de la misma, han incrementado sobremanera el interés por los datos referentes a la mortalidad en España, según el Instituto Nacional de Estadística ${ }^{2}$

Para conocer la magnitud del problema en población general y el estado actual de la cuestión puede servirnos de referencia unos datos de nuestro entorno: en el año 2009 se produjeron en España 383.486 fallecimientos, un $0,7 \%$ menos que en 2008 . La tasa bruta de mortalidad disminuyó hasta situarse en 8,35 fallecidos por cada mil habitantes, frente a los 8,43 del año 2008. Por zonas y comunidades autónomas en lo referente a la mortalidad, la mitad noroeste peninsular presentó, en términos relativos, un mayor número de defunciones en 2009. Cabe mencionar en este sentido que esta zona presenta poblaciones más envejecidas que el resto de España. Así, las tasas de mortalidad más elevadas se dieron en Principado de Asturias (con 12,1 defunciones por cada mil habitantes), Galicia $(11,0)$ y Castilla y León $(10,7)^{2}$

En la literatura científica se muestra la asociación que tiene estas pérdidas con determinados trastornos, la depresión en los viudos/as ${ }^{3}$ casi la mitad presentan ansiedad o crisis de angustia ${ }^{4}$; existe abuso de alcohol ${ }^{5}$ y la utilización de psicofármacos en los 18 primeros meses $^{6}$ en definitiva se puede llegar a desarrollar un duelo patológico ${ }^{7}$ incrementándose el riesgo de muerte (principalmente por eventos cardiacos y suicidio), teniendo los viudos un alto porcentaje de probabilidades de morir prematuramente durante el primer año ${ }^{8}$. por otro lado, la tasa promedio anual de consultas es un $80 \%$ mayor en los dolientes ${ }^{9}$ y el nivel asistencial ideal para sus cuidados es, según algunos autores, la atención en primera instancia o la prevención y consejo primario ${ }^{10-12}$.

De este modo en algunos casos la relación entre procesos de duelo y psicopatología es estrecha. Los duelos acumulados y/o insuficientemente elaborados, los acontecimientos vitales estresantes, favorecen el desequilibrio mental, la psicopatología, aunque el cúmulo de variables que intervienen hace difícil el hallazgo de relaciones causales.

Distintos autores proponen el duelo patológico como una entidad clínica diferente, intentando clasificar la sintomatología como un síndrome e incluso haciendo propuestas de criterios para su clasificación tal como Zisook ${ }^{13}$ y Horowitz ${ }^{14}$, desde el modelo del trastorno de estrés postraumático, o Prigerson ${ }^{15}$ y Raphael ${ }^{16}$, basándose en criterios más estadísticos. Los riesgos y ventajas de tal intento han sido revisados por diferentes autores y desde diferentes ámbitos ${ }^{17}$.

En los manuales de diagnostico de enfermedades mentales la clasificación de los duelos problemáticos se establece en el apartado de otros problemas que pueden ser objeto de atención clínica, y dentro de este en los problemas adicionales que pueden ser objeto de atención clínica «Z63.4 Duelo [V62.82]», DSM IV-TR.

De todas formas hay que tener en cuenta que es obvio que el duelo como en gran parte de las manifestaciones psicológicas, lo que se define por normalidad o anormalidad depende de evaluaciones culturales.

Hay muchas variables que se han relacionado con los procesos de duelo para diferenciar a los dolientes, en relación al género se ha encontrado grandes diferencias entre ambos géneros en el sentido de una mayoría de consultas por estos motivos fueron realizadas por mujeres ${ }^{18}$. La mayor demanda de mujeres en los procesos de duelo es coincidente con todos los hallazgos de la literatura y oscila entre el 60 y el $80 \%$ en literatura anglosajona cuando incluyen todo tipo de parentesco ${ }^{19,20}$ llegando hasta el $95 \%$ de mujeres ${ }^{20}$. También en las mujeres se manifestaría mayor malestar inicial que en el varón, el ajuste psicológico y social sería similar en ambos, mientras ellos presentaban mayores dificultades de recuperación a los dos o cuatro años, e incluso, aumento de mortalidad cardiovascular en algunos grupos de edad, el riesgo de síntomas psiquiátricos en mujeres que perdían a sus maridos era 7 veces superior a lo esperado, mientras en varones la pérdida de la esposa aumentaba 4 veces más el riesgo para ese grupo de edad ${ }^{21,22}$.

W. y M. Estroebe ${ }^{23}$, señalan que son las mujeres las que tienen un mayor número de consultas por la pérdida de seres queridos, realizan más conductas de lloros y estas duran más tiempo, toman más cantidad de psicofármacos que los hombres, entre otras variables. El Harvard Study citado en Tizón ${ }^{24}$ donde se analizó la conducta de 49 viudas, se concluyó que estas tenían un mayor desajuste al principio del proceso de duelo y este disminuía con respecto a los viudos trascurrido unos años

Se ha especulado sobre la posibilidad de que las mujeres pueden experimentar un duelo distinto y con diferentes resultados porque reciben más apoyo social que los hombres. Un sólido estudio de Stroebe $^{23}$ y sus colegas indica que no es así. Bowlby ${ }^{21}$, señala la estructura de la personalidad del deudo cuando se intenta entender su respuesta ante la pérdida.

Otro de los factores de importancia en los procesos de duelo es la forma fallecimiento de la persona querida. Tizón ${ }^{24}$ considera que hay una serie de circunstancias que hacen el proceso de duelo más complicado, tales como las muertes inesperadas.

En la literatura anglosajona se utiliza el acrónimo $\mathrm{NASH}^{25}$, cuyas silabas señalan cómo se ha producido la muerte de la persona querida: Muerte Natural, por Accidente, por Suicidio u Homicidio. Esto se ha utilizado para proporcionar una idea de la gravedad y las complicaciones del duelo, asociado a cómo se ha producido la muerte, en intensidad creciente que va desde la muerte por causas naturales, o por accidente, hasta la muerte como resultado de un suicidio u homicidio.

Las consecuencias psicológicas para las personas que han perdido a su ser querido son múltiples, las familias de los fallecidos por suicidio a menudo se avergüenzan de sí mismas, y en muchas culturas, son estigmatizadas socialmente.

Cuando una muerte se considera evitable, aparecen cuestiones relacionadas con la culpa. Las muertes evitables suelen estar asociadas a litigios prolongados y ello puede alargar el proceso de duelo de las personas implicadas ${ }^{26}$.

En estas y otras circunstancias, parece adecuado que evaluar el proceso de duelo es una cuestión fundamental, sobre todo en los primeros momentos donde es necesario saber si una persona está procesando un duelo normal o patológico o tiene riesgo de sufrirlo, si con sus potencialidades y apoyos sociales es suficiente o por el contrario es necesario prestarle ayuda especializada derivándolo a un servicio psicológico o de salud mental en general.

Se han revisado algunos de los cuestionarios de duelo publica$\operatorname{dos}^{27-29}$ y se ha seleccionado el Inventario Texas Revisado de Duelo. ITRD es el acrónimo del Inventario Texas Revisado de Duelo, versión adaptada al castellano del Texas Revised Inventory of Grief de Faschingbauer y cols. ${ }^{30,31}$ Es de muy fácil aplicación y puede 
puntuase directamente sin la utilización de plantillas ni material adicional, solamente con «lápiz y papel», tiene unas adecuadas propiedades psicométricas y es una prueba muy utilizada.

En los estudios publicados en España se han utilizado muestras de dolientes viudos y viudas con una edad bastante avanzada ${ }^{32-35}$, con lo cual los resultados de los mismos se pueden generalizar para este grupo de edad, quedando un vacío en cuanto a la investigación y valoración de posibles duelos complicados en población española adultos jóvenes y en población general.

En el ámbito de las Fuerzas Armadas en general hay una especial preocupación por la atención a las víctimas y a las personas que han perdido a sus seres queridos en actos de servicio, es de justicia reconocer el sacrificio de aquellos que fallecen o resultan heridos en acto de servicio, así como el de sus familias y compañeros lo que conlleva la obligación de velar por la mejor y más rápida recuperación integral de los heridos y de apoyar y amparar en todo lo posible a los que han perdido a uno de sus seres queridos, teniendo en cuenta que se trata de hechos que originan graves consecuencias de todo orden para los afectados y sus familias, acreedores de un especial apoyo y calor humano, no sólo con carácter inmediato, sino también en momentos posteriores. Esto queda reflejado en normas específicas y normalizaciones como los STANAG ${ }^{36}$ de referencia. En las Fuerzas Armadas Españolas, la atención psicológica y de la sanidad militar en general se extiende a los profesionales de las mismas como a sus familiares, en el año 2004 se publicó el Real Decreto 2394/2004 ${ }^{37}$, por el que se aprobaba el Protocolo para la recuperación, identificación, traslado e inhumación de los restos mortales de los fallecidos en operaciones fuera del territorio nacional, que a su vez señala los apoyos que deben tener los familiares, desde la sanidad militar (psicología y medicina). Recientemente se ha publicado la Orden Ministerial $71 / 2010^{38}$ por la que se crea la Unidad de apoyo a heridos y familiares de fallecidos y heridos en acto de servicio de las Fuerzas Armadas, quedando de esta forma patente la especial preocupación por estos temas. Como suplemento técnico de esta normativa y consecuencia de la experiencia acumulada en el trabajo diario, parece importante poder realizar una medida objetiva del proceso de duelo en los familiares, compañeros y demás personas que han perdido a un ser querido, con esta intención se realizó el presente estudio.

\section{OBJETIVO}

El objetivo del estudio es conseguir valorar psicométricamente el Inventario Texas Revisado de Duelo (ITRD), versión adaptada al castellano del Texas Revised Inventory of Grief de Faschingbauer y cols. ${ }^{30,31}$, adaptado en España por García y cols ${ }^{35}$. Con una muestra de adultos jóvenes pertenecientes a las Fuerzas Armadas Españolas, estudiando si los datos se adaptan a lo descrito en la literatura.

\section{MATERIAL Y MÉTODO}

\section{Muestra}

Se ha utilizado una muestra de adultos jóvenes militares, que se encontraban en periodo de formación en el Ejército del Aire, la participación fue voluntaria y anónima respondiendo al cuestionario pro-
Tabla 1. Cómo fue el suceso

\begin{tabular}{lcc}
\hline \multicolumn{1}{c}{ Suceso } & Frecuencia & Porcentaje \\
\hline Suicidio & 6 & 4,3 \\
Accidente & 26 & 18,4 \\
Natural & 97 & 68,8 \\
Otras & 12 & 8,5 \\
Total & 141 & 100,0 \\
\hline
\end{tabular}

Tabla 2. Matriz de componentes rotados

\begin{tabular}{lcc}
\hline & \multicolumn{2}{c}{ Componentes Rotados } \\
& Duelo actual & Duelo agudo \\
\cline { 2 - 3 } Item 1 & 0,072 & 0,805 \\
Item 2 & 0,160 & 0,790 \\
Item 3 & 0,123 & 0,718 \\
Item 4 & 0,366 & 0,408 \\
Item 5 & 0,297 & 0,636 \\
Item 6 & 0,310 & 0,767 \\
Item 7 & 0,296 & 0,503 \\
Item 8 & 0,253 & 0,749 \\
Item 9 & 0,730 & 0,326 \\
Item 10 & 0,675 & 0,218 \\
Item 11 & 0,542 & 0,183 \\
Item 12 & 0,666 & 0,110 \\
Item 13 & 0,690 & 0,313 \\
Item 14 & 0,688 & 0,231 \\
Item 15 & 0,554 & 0,280 \\
Item 16 & 0,455 & 0,119 \\
Item 17 & 0,621 & 0,222 \\
Item 18 & 0,584 & $-0,026$ \\
Item 19 & 0,437 & 0,330 \\
Item 20 & 0,545 & 0,191 \\
Item 21 & 0,679 & 0,279 \\
\hline
\end{tabular}

Método de extracción: Análisis de componentes principales. Método de rotación: Normalización Varimax con Kaiser.

Tabla 3. Baremos: Percentiles y Cuartiles.

\begin{tabular}{lcccc}
\hline \multirow{2}{*}{ N Válidos } & & D. Agudo & D. Actual & D. Total \\
\hline Percentiles & 10 & 9,00 & 141 & 141 \\
\hline & 20 & 10,40 & 19,00 & 30,00 \\
& 25 & 11,00 & 23,00 & 35,00 \\
& 30 & 12,00 & 27,00 & 37,00 \\
& 40 & 14,00 & 30,00 & 40,00 \\
& 50 & 16,00 & 32,00 & 45,00 \\
& 60 & 18,00 & 36,20 & 54,00 \\
& 70 & 21,00 & 40,00 & 61,00 \\
& 75 & 22,00 & 40,50 & 62,00 \\
& 80 & 23,00 & 42,00 & 63,60 \\
90 & 27,00 & 48,00 & 69,00 \\
99 & 35,16 & 62,48 & 92,96 \\
\hline
\end{tabular}


Tabla 4. Descriptivos

\begin{tabular}{|c|c|c|c|c|c|c|}
\hline \multirow{5}{*}{ D. Agudo } & & \multirow[t]{2}{*}{$\mathbf{N}$} & \multirow[t]{2}{*}{ Media } & \multirow[t]{2}{*}{ Desviación típica } & \multicolumn{2}{|c|}{ Intervalo de confianza para la media al $95 \%$} \\
\hline & & & & & Límite inferior & Límite superior \\
\hline & varón & 105 & 16,53 & 6,907 & 15,20 & 17,87 \\
\hline & mujer & 36 & 18,44 & 7,093 & 16,04 & 20,84 \\
\hline & Total & 141 & 17,02 & 6,980 & 15,86 & 18,18 \\
\hline \multirow[t]{3}{*}{ D. Actual } & varón & 105 & 31,98 & 10,308 & 29,99 & 33,98 \\
\hline & mujer & 36 & 37,08 & 11,108 & 33,32 & 40,84 \\
\hline & Total & 141 & 33,28 & 10,713 & 31,50 & 35,07 \\
\hline \multirow[t]{3}{*}{ D. Total } & varón & 105 & 48,51 & 15,251 & 45,56 & 51,47 \\
\hline & mujer & 36 & 55,53 & 16,882 & 49,82 & 61,24 \\
\hline & Total & 141 & 50,30 & 15,920 & 47,65 & 52,96 \\
\hline
\end{tabular}

Tabla 5. Fiabilidad.

\begin{tabular}{lccc}
\hline Alfa Cronbach & Faschingbauer 1981 & García 1996 & Samper \\
\hline Agudo, pasado & 0.77 & 0.75 & 0,86 \\
Actal, Presente & 0.86 & 0.86 & 0,879 \\
Total & no & no & 0,911 \\
\hline
\end{tabular}

puesto en caso de haber sufrido la pérdida de una persona querida. La muestra fue de 141 sujetos, con mayor número de varones $(74,5 \%)$ que de mujeres $(25,5 \%)$, porcentaje superior a la representación de la mujer en el conjunto de las Fuerzas Armadas Españolas ${ }^{39}$

Se les comunicó a los participantes la intención de esta investigación y se les ha sugerido su participación en la misma de forma consentida.

Se marcaron unos criterios de inclusión con el fin de conseguir una muestra válida para el estudio, como la participación voluntaria, haber perdido a un ser querido, y que desde esta muerte hubiera transcurrido más de 1 mes (al principio el doliente está bloqueado) y menos de 3 años (hacia los 3 años el duelo se va estabilizando) $)^{32}$ desde la muerte de la persona querida.

Como criterios de exclusión se tuvo en cuenta que la muestra había sido previamente seleccionada, pasando unas pruebas de selección para ingreso en las Fuerzas Armadas, en las que se eliminan por selección negativa los candidatos con posible deterioro mental o físico, y se eligen con un nivel intelectual adecuado, dentro de los criterios de normalidad, CI $>80$.

\section{Procedimiento}

El procedimiento seguido para recoger los datos fue a través del cuestionario confeccionado al efecto, donde recogieron los ítems de la prueba ITRD., y el cuestionario de datos personales.

Para recoger las contestaciones de los sujetos se ha diseñado una hoja de lectora óptica, mediante la cual se realizará la lectura y explotación estadística de los datos, con un paquete informático de análisis estadístico SPSS 12.0 y con la hoja de cálculo Excel, de Microsoft.

\section{Descripción de la prueba utilizada}

El inventario Texas Revisado de Duelo (ITRD) de Faschinbaguer y cols ${ }^{30,31}$ se elaboró en una población amplia y representativa de dolientes de EEUU ( $\mathrm{N}=260$ en un trabajo inicial y 328 en otro estudio replicación del primero), a partir de una versión ampliada de 59 ítems que incluía los 7 del Texas inicial. En esta ocasión, para la selección de los ítems se utilizó el análisis factorial; del análisis resultaron relevantes dos factores y se eliminaron todos los ítems que no saturaban 0,40 o más a cualquiera de ellos; quedando los 21 definitivos, agrupados, 8 en la parte I y 13 en la parte II.

La prueba en la actualidad consta 21 ítems con 5 categorías de respuesta (tipo Likert) -desde «completamente verdadera» (5 puntos) hasta «completamente falso» (1 punto)-, es autoadministrado y se rellena aproximadamente en 10 minutos. Para su evaluación se suman las puntuaciones de cada ítem y se calculan los totales de cada una de las dos partes del cuestionario. La primera parte de 8 ítems referida a la conducta y los sentimientos del doliente en los momentos posteriores cercanos a la muerte de la persona querida, denominada parte I (duelo agudo), cuya puntuación oscila entre 8 y 40; y la otra de 13 ítems, que hace referencia a los sentimientos actuales, denominada parte II (duelo actual), cuya puntuación oscila entre 13 y 65. Según sus autores permite agrupar a los dolientes en cuatro categorías: Ausencia de duelo, duelo retardado, prolongado y agudo. Es igualmente sensible a los cambios en el tiempo, tiene estudios de fiabilidad y validez, y al menos existen una versión en hispano ${ }^{39}$ y otra en francés ${ }^{40}$ además de la original. La prueba se completa con una puntuación total de la escala que se ha denominado duelo total, siendo el sumatorio de las partes descritas anteriormente.

A esta prueba se ha añadido un breve cuestionario de datos biográficos para recoger la información, necesaria para la demostración de las hipótesis propuestas en la literatura en los estudios realizados con la prueba, donde se pregunta sobre la edad, el sexo, la forma de fallecimiento, el tiempo trascurrido desde la perdida de la persona querida

La edad de los sujetos en un $97.9 \%$ estuvo en el rango entre los 18 y los 30 años con 3 sujetos que superaron esta edad, que se dejaron en el estudio por estimar que no distorsionarían los resultados y sí añadirían datos con valor estadístico al estudio en general. 
Tabla 6. Comparación.

\begin{tabular}{|c|c|c|c|c|c|c|c|c|c|c|}
\hline & \multicolumn{2}{|c|}{ García 1996} & \multicolumn{2}{|c|}{$\begin{array}{c}\text { Faschingbauer } \\
1981\end{array}$} & \multicolumn{4}{|c|}{$\begin{array}{l}\text { Grabowki y Frantz } \\
\text { L. latinos y A. anglos }\end{array}$} & \multicolumn{2}{|c|}{ Samper } \\
\hline & Media & Desv. & Media & Desv. & L. med & $\mathrm{L}$ desv & A med & A desv & Media & Desv. \\
\hline D. agudo & 26.25 & 7,34 & 17,8 & 8,63 & 22,4 & 10 & 20.1 & 8,7 & 17,2 & 6,98 \\
\hline D. actual & 51,91 & 10,21 & 37,1 & 16,73 & 49,2 & 12 & 39,6 & 14,3 & 33,28 & 10,71 \\
\hline D. total & & & & & & & & & 50,3 & 15.92 \\
\hline
\end{tabular}

Tabla 7. Distribución con referencia a percentil 50.

\begin{tabular}{ccc}
\hline $\begin{array}{c}\text { Duelo agudo } \\
\text { P50 (16) }\end{array}$ & $\begin{array}{c}\text { Duelo actual } \\
\text { P50 (32) }\end{array}$ & Resultado \\
\hline Alto & Alto & D. Prolongado \\
Alto & Bajo & D. Resuelto \\
Bajo & Bajo & D. Ausente \\
Bajo & Alto & D. Retrasado \\
\hline
\end{tabular}

\section{RESULTADOS}

\section{Diferencias en el proceso de duelo y en la forma del proceso}

En este apartado se utilizó lo descrito en la literatura ${ }^{25}$ Haciendo referencia a cómo fue el fallecimiento de la persona querida: De forma natural, por accidente, por suicidio o por homicidio, este último apartado no tuvo ninguna repercusión no existiendo ningún fallecimiento por homicidio en la muestra estudiada, la mayoría de los fallecimientos y siguiendo con la argumentación realizada anteriormente, se produjo de forma natural en un $68.8 \%$, seguido por los accidentes con un $18,4 \%$ y a mayor distancia por los suicidios con $4,3 \%$, No se observó diferencias significativas en el análisis de esta variable.

Se puede observar las medidas de tendencia central y dispersión de varones y mujeres, en duelo agudo, actual y total, observándose que la medida de los varones en todos los casos quedó por debajo de la medida de las mujeres, las diferencias fueron significativas en las medidas de duelo en el momento de realizar la prueba, «duelo actual» y en la medida total de la prueba, «duelo total» $(\mathrm{P}<0.05)$.

Se observan las medidas de la prueba utilizada con diferentes muestras: La adaptación al castellano de García ${ }^{35}$, (viudos/ as, $n=118$, tiempo desde la pérdida entre 3 meses y 3 años). La muestra original utilizada por Faschingbauer ${ }^{30}$ (todo tipo de duelo, $\mathrm{n}=295$, tiempo desde la pérdida entre 1 y 5 años). Y la versión en muestra de hispanos y anglos de Grabowski \& Frantz ${ }^{40}(n=100)$. Y el estudio actual (adultos jóvenes, $n=141$ ).

Las medidas de la prueba observadas en este estudio están más próximas a las medidas tomadas en el estudio original de la prueba de Faschingbauer ${ }^{31}$ e incluso a la muestra de angloparlantes de Grabwski y Frantz ${ }^{40}$.

En este estudio se ha observado la recomendación de los autores originales de la prueba respecto a la diferenciación de duelos complicados: El autor en el manual de la prueba ${ }^{31}$ hace una distribución de los afectados con respecto a su puntuación, diferenciándolos según se puede ver en la tabla 7 . La denominación con relación a la puntuación en duelo agudo y duelo actual por encima
Tabla 8. Tipo de duelo adaptado a la versión original Faschingbauer percentil 50.

\begin{tabular}{lcc}
\hline & Frecuencia & Porcentaje \\
\hline 1 Ausente & 1 & 0,7 \\
2 Retrasado & 5 & 3,5 \\
3 Resuelto & 7 & 5,0 \\
4 Prolongado & 128 & 90,8 \\
Total & 141 & 100,0 \\
\hline
\end{tabular}

(Alto) o por debajo (Bajo) del percentil 50 (P50) de la prueba. Con esta distribución de puntuaciones se obtienen cuatro tipos de duelo: Duelo prolongado, es aquel sujeto que puntúa por encima de P50 en ambos momentos (Alto-Alto). Duelo resuelto, el sujeto que puntúa por encima de P50 en duelo agudo (Alto-Bajo). Duelo ausente, aquel sujeto que puntúa por debajo del P50 en ambos casos (BajoBajo). Y por último el duelo retrasado corresponde al sujeto que puntúa por encima del P50 en el factor duelo actual (Bajo-Alto).

En la tabla 8 se puede observar la distribución de las puntuaciones obtenidas en este estudio, de esta forma se obtendría un $90.8 \%$ de sujetos clasificados como duelo prolongado, en oposición a una sola persona que puntuaría como duelo ausente $0.7 \%$.

En una variación para clasificar a las personas evaluadas en este estudio, se ha tomado como referencia el percentil 99 (P99), elegido por consenso de expertos para este estudio por ser utilizado en la práctica del trabajo psicológico en las evaluaciones con muestras de población no clínica. Así se observa que únicamente una persona sobrepasa el P99 en los tres factores tenidos en cuenta en este estudio: Mujer con edad entre 18 y 31 años, con un proceso de duelo por la pérdida de un amigo de forma natural, por enfermedad grave, sin ningún tratamiento en salud mental.

\section{Adaptación psicométrica del instrumento.Distribución de las puntuaciones y Baremos}

La escala mostró unos índices estadísticos adecuados (Kaiser Meyer Olkin , 0.878), para su factorización y (komogorov Smirnov) para su distribución normalizada, con lo que se podrá realizar los análisis con garantía estadística.

Se ha realizado un estudio de la carga factorial en la prueba, según se aprecia en la tabla 2, para ello se utilizó el método de factorización de análisis de componentes principales, varimáx con káiser, ajustado al estudio de los factores de duelo actual y duelo agudo, según indica la misma prueba, obteniéndose una distribución de las cargas similar a lo encontrado en otros estudios ya cita$\operatorname{dos}^{35}$, y en la versión original de Faschingbauer ${ }^{31}$ 
Se ha observado en los datos de factorización, que los ítems: 4 , [factor $1(0.366)$ y factor $2(0.408)$ ], y el ítem 19 , [factor $1(0.437)$ y factor $2(0.330)]$. Tenían cargas factoriales más aproximadas que otros ítems, en los dos factores, aunque la carga mayor era según lo esperado.

Para el análisis de la distribución de las puntuaciones en la escala se ha obtenido los percentiles, en ellas se ha observado las puntuaciones correspondientes a los percentiles para 11 grupos, incluyendo los cuartiles y el percentil 99, que se tomará como referencia de los duelos posiblemente complicados para población no clínica.

En el análisis de la fiabilidad de la prueba se observó unos índices de consistencia interna similares a los encontrados por otros autores tanto con muestras Españolas (García y cols ${ }^{35}$, viudos/ as, $\mathrm{n}=118$ ) como con muestras Internacionales (Faschingbauer y cols $^{30,31}$, todo tipo de duelo, $\mathrm{n}=260$ ), se encontró unos índices de consistencia interna (alfa de Cronbach) adecuados por encima de lo esperado (Alfa>.07) en todas las escalas, y superior en todos los casos a lo obtenido en los otros estudios.

En cuanto al índice de consistencia interna obtenido en la escala total no había datos de los otros estudios, pero se estima que podría estar en la misma dirección que lo observado en las subescalas. En el caso que nos ocupa se obtuvo un índice en la escala total alto $($ Alfa $=0.911)$.

\section{Análisis de la diferencias en el proceso de duelo}

Tras la realización del estudio trasversal de los 141 sujetos adultos jóvenes, se ha observado algunas diferencias entre este estudio y algunos otros realizados con el mismo cuestionario y con diferente muestra en España y otros países.

La muestra es diferente a la encontrada en otros estudios con población Española, en los que la edad era ya avanzada, viudos y viudas que habían perdido a su pareja en los últimos estadios de la vida ${ }^{32}$.

También se han utilizado en otros trabajos como los de Olmeda $\mathrm{MS}^{18}$, un número similar de casos $(\mathrm{n}=150)$, pero seleccionados en una muestra que ya había consultado por tener problemas en la elaboración del duelo, que se denominan: «Población psiquiátrica ambulatoria». Siendo el rango de edad en este caso desde los 18 a los 82 años en el momento de la consulta, un rango muy amplio. En este caso considerando la muestra como población psiquiátrica, se espera una prevalencia del $25 \%$ de formas complicadas de duelo contrastado con la población general en que la prevalencia oscila entre el 4 al $6 \%$ según Clayton ${ }^{19}$.

Se considera que una de las primeras aportaciones de este estudio es la muestra analizada joven adulta, y correspondiente a personal de las Fuerzas Armadas Españolas.

\section{Variable género}

En relación con la variable género, se han encontrado diferencias de medias en las dos subescalas y en la medida de duelo total, siendo mayores las puntuaciones observadas en mujeres que en hombres.

Se puede apreciar en los datos que son las mujeres las que muestran mayor puntuación, infiriendo por tanto que pueden tener más dificultades para superar el proceso de duelo. Esto entra en contradicción con otros estudios que mantienen una postura opuesta (Harvard study) ${ }^{24}$.
En los momentos cercanos a la pérdida las personas sufren una intensa emocionalidad y malestar, que tanto hombres como mujeres experimentan de forma similar. El perder a alguien querido, supone un sufrimiento igual para hombres que para mujeres, sin embargo pasado ese período inicial y posiblemente por las influencias culturales y de socialización, las mujeres muestran mayor dificultad para procesar esta dificultad. Este hecho puede estar relacionado con la dificultad mayor del varón para mostrar sus sentimientos, y puede que por este mismo motivo las mujeres consulten más que los hombres como señala Olmedo $\mathrm{MS}^{18}$. Lo que sí parece claro es que los hombres y las mujeres, tienen diferente forma de procesar el sufrimiento que provoca la pérdida de seres queridos, y que son las mujeres las que muestran mayores dificultades en comparación a los hombres, que parecen mostrar mejor ajuste psicológico.

Sería muy interesante para posteriores estudios analizar el sufrimiento de la persona y su expresión con relación a los valores culturales y sociales, relacionándolo con la variable género.

\section{Forma del suceso}

La forma de producirse el fallecimiento e intentar explicar la relación entre este hecho y la medida de duelo observadas, ha sido un tema de interés en los estudios revisados, señalándose este hecho como uno de los factores de riesgo para la elaboración personal de los procesos de duelo ${ }^{20}$.

Las diferencias encontradas en este estudio no fueron significativas. No parece que la forma del suceso tenga valores discriminativos en este estudio. Una observación de los datos muestra que los fallecimientos producidos por accidentes tienen mayores consecuencias a lo largo del tiempo que otros sucesos, pudiendo provocar secuelas más traumáticas, aunque no son significativas las diferencias observadas, si sería de interés para posteriores estudios.

\section{Estudio de la prueba: Inventario de Texas revisado de duelo (ITRD)}

Los ítemes de la escala total se distribuyeron según especificaba la versión original, la medida de total de la prueba «duelo total» quedaba con una influencia mayor de la subescala duelo actual, en un $62 \%$ frente a un $38 \%$ de carga de la subescala de duelo agudo, este es uno de los motivos que ha hecho que se estudien las dos subescalas y la escala total de forma independiente, a la vez que se pueden obtener más conclusiones realizándolo de esta forma.

Los ítemes de la prueba se distribuían en los dos factores rotados, de forma similar a lo encontrado en otros estudios de García ya citados, y en la versión original de Faschingbauer ${ }^{31}$, se puede decir que la distribución de los ítemes fue adecuada en la muestra de jóvenes adultos, igual que lo observado en la literatura.

Algunos íitemes requieren un estudio más en profundidad. El ítem 4 (A veces le/la echo mucho de menos), correspondiente a la primera parte de la prueba, tiene una redacción diferente al resto, no incluye el preámbulo «Tras su muerte...», ni marca el momento temporal a qué se refiere, esto puede ser causa de que el ítem no tenga la definición factorial tan clara como el resto de ítemes de la primera parte. Las personas que pierden a un ser querido, a lo largo del tiempo asimilan tales perdidas integrándolas como parte de su vida, sin embargo si son preguntados sobre si echan de menos a su ser querido seguramente a veces le echan de menos, con lo cual este ítem que en principio, eva- 
lúa el sentimiento de echar de menos a la persona querida, es un buen predictor del proceso de duelo, pero requiere de una evaluación más en profundidad y quizás de una nueva redacción. Algo similar ocurre con el ítem 19 (Las cosas y las personas que le rodean todavía me hacen recordarle/la) de la segunda parte de la prueba, evalúa el posible condicionamiento de cosas y personas que estaban asociadas antes y siguen asociadas ahora a la persona querida.

Por ello se cree que una revisión y un estudio en profundidad de los ítemes en diferentes muestras de población o en población general serían muy adecuados para ir ajustando la prueba.

Una aplicación de esta escala podría ser detectar posibles duelos complicados, para esto es necesario realizar la distribución de las puntuaciones de los sujetos y baremar los resultados de la misma. En este estudio se hace un estudio de autobaremo de los 141 sujetos, observándose la distribución de las puntuaciones obtenidas y su distribución en percentiles, se ha estimado en este estudio que es importante el percentil 99 (P99), que se estima como índice de corte importante para detectar posibles duelos complicados, dado que la población de estudio es normal no clínica.

Tomando como referencia este percentil (P99) se establecen los límites en los que los deudos podrían estar procesando un duelo problemático, que en principio sería necesario revisar por un servicio especializado de psicología o psiquiatría. Estas medidas quedan fijadas en la primera parte «duelo agudo» en 35,16 puntos, en la segunda parte «duelo actual en 62,48 puntos, y en la medida de la escala total en 92,96 puntos.

Este aporte estimado difiere de lo que aconsejan los autores originales de la prueba, Faschingbauer y $\mathrm{col}^{31}$., que fijan el punto de corte en el percentil 50 (P50), y diferencian 4 grupos de duelo diferentes: Ausencia de duelo, duelo retrasado, prolongado, y resuelto. Tal como se observa en este estudio (tablas 7 y 8 ), un $90.8 \%$ de sujetos estarían clasificados como duelo prolongado y un $3.5 \%$ de sujetos estarían clasificados como un duelo retrasado, que pueden ser los procesos de duelo con problemas. Se puede inferir por tanto que son 133 sujetos los que pueden necesitar una revisión de caso, frente a únicamente 8 que han resuelto su proceso de duelo de forma adecuada (duelo ausente y duelo resuelto)

En contraposición, en la propuesta que se realiza en este estudio clasificando a los sujetos con un percentil de 99 (P99), se observaría un solo caso que podría necesitar atención especializada para superar su proceso de duelo. Siendo el perfil de este sujeto el que se describe: «Mujer con edad entre 18 y 30 años, con un proceso de duelo por la pérdida de un amigo por enfermedad grave, sin ningún tratamiento en salud mental, en el momento de realizar la evaluación». Queda para posteriores estudio con poblaciones diferentes y diferentes muestras clínicas perfilar el punto de corte ideal para identificar personas con posibles duelos complicados.

\section{DISCUSIÓN}

En los estudios realizados, se tomó de referencia el estudio de Garciá y $\operatorname{cols}^{32}$, y el realizado con la versión original de Faschingbauer y cols ${ }^{31}$ y otra realizada con una muestra de Anglosajones de Grabwski y Frantz ${ }^{40}$ para los análisis comparativos de la prueba.

Tras los estudios realizados del inventario de Texas, al compararlos con los estudios de García y cols ${ }^{33}$ se encontraron algunas diferencias observándose:
Que la carga factorial en los dos factores obtenidos a través de la Matriz rotada de los ítems, era similar, obteniéndose unos índices de factorización en su mayor parte superiores a los del estudio de García y $\operatorname{cols}^{33-35}$.

En el análisis comparativo de la fiabilidad del tests con muestras Españolas de García y cols ${ }^{35}$ y muestras Internacionales de la prueba original de Faschingbauer y cols ${ }^{31}$, se encontró unos índices (Alfa de Cronbach) adecuados de la escala, por encima de lo esperado (Alfa>.07) en todas las escalas, y superior en todos los casos a lo obtenido en los otros estudios.

En cuanto al índice obtenido en la escala total no había datos de los otros estudios, pero se estima que estará en la misma dirección que las subescalas, de duelo agudo y actual. En el caso que nos ocupa se obtuvo un índice muy importante (Alfa $=0.911)$.

En las medias de la prueba obtenidas en este estudio se observa que son similares a las obtenidas en la prueba original se Faschingbauer y cols ${ }^{31}$, incluso con unos índices de dispersión menores. Esto podría entra en contradicción a lo mencionado por García y $\operatorname{cols}^{33}$, en el sentido de que en las muestras de población hispana, por una posible aproximación cultural a la población Española, se espera obtener son unos resultados similares. No encontrándose confirmación en este estudio, en el que se observa más aproximación a muestras originales de Faschingbauer y $\operatorname{cols}^{31}$ y a muestras de Anglosajones de Grabwski y Frantz ${ }^{40}$.

Este hecho se puede interpretar por las diferencias en las muestras utilizadas, dado que en todos los caso son muestras clínicas, menos en el trabajo que nos ocupa y por ello se observa medidas de duelo, menos elevadas, que en estudios con población clínica.

También hay que tener en cuenta que al ser una muestra de población joven, existe una influencia tecnológica y cultural anglosajona importante, que la diferencia de otras muestras de más edad en las que esta influencia es menor.

Por todo lo explicado hasta este momento sería interesante contar con un número mayor de estudios e investigación en esta área tan importante y de tanta transcendencia en todas las culturas y en todos los tiempos.

\section{CONCLUSIÓN}

Con este trabajo de interés para el enriquecimiento de los instrumentos de diagnóstico psicológico, de interés para ofrecer una atención más especializada en momentos difíciles para familiares, compañeros y amigos que han perdido a un ser querido, añadiendo un pequeño grano de arena a los estudios sobre el duelo y cómo medir este constructo. Quizás estos estudios ayuden para realizar una prevención eficaz y efectiva en las victimas o para detectar posibles procesos de duelo complicados antes de que lleguen a producir más problemas a los deudos.

Se ha realizado una propuesta de clasificación de los procesos de duelo según la versión original de Faschingbauer y cols ${ }^{31}$ y según los resultados obtenidos por este estudio que por supuesto requiere de más investigación y estudio, pero que se pueden tomar como referencia para posteriores trabajos.

Por este motivo parece necesaria la evaluación y estudio de pruebas tan especificas, dando a la vez un aporte más científico a sufrimientos que a pesar de acompañar a la condición humana desde siempre, cada vez demandan más atención en nuestras sociedades. 
Este estudio se concluye con las siguientes afirmaciones:

- La prueba muestra análisis estadísticos adecuados, similares a los obtenidos en los estudios originales y los realizados en España, por lo que se considera una prueba psicométricamente adecuada para la medida del proceso psicológico por la pérdida de seres queridos, en una muestra de personal de las Fuerzas Armadas Españolas jóvenes adultos.

- Algunos ítems de la prueba pueden requerir un análisis y revisión para mejorar la prueba y su aplicación en todo tipo de población.

- La intensidad del duelo es mayor en mujeres que en hombres, y esta diferencia se incrementa con el paso del tiempo.

- La forma en que se produce la pérdida parece una variable importante en la literatura y requiere más investigación, en este estudio las diferencias no han sido significativas. Las pérdidas por accidentes pueden dejar más secuelas que otras pérdidas en este estudio.

- Se requiere una mayor investigación y estudio para obtener medidas y baremos adecuados del proceso de duelo, en población joven y general española no clínica.

- Parece que el perfil más sobresaliente (a pesar de la poca representación de las mujeres en este estudio) en cuanto a intensidad del proceso por la pérdida de seres queridos es de una mujer, sin ningún tratamiento en salud mental, y habiendo perdido a un ser querido por enfermedad.

\section{BIBLIOGRAFÍA}

1. Holmes TH, Rahe RH. The Social Readjustment Rating Scale. J Psychosom Res 1967; 11: 213-8.

2. Instituto Nacional de Estadística (www.ine.es).

3. Zisook S, Shuchter SR.Depression through the first year after the death of a spouse. Am J Psychiatry 1991; 148:1346-52.

4. Jacobs S, Hansen F, Kasl S, Ostfeld A, Berkman I, Kim K. Anxiety disorders during acute bereavement: risk and risk factors. J Clin Psychiatry 1990; 51: 269-74.

5. Maddison D, Viola A. The health of the widows in the year following bereavement. J Psychosom Res 1968; 12: 297-306.

6. Parkes CM. Effects of bereavement on physical and mental health-a study of the medical records of widows. Brit Med J 1964; 274-9.

7. Jacobs S. Traumatic Grief: Diagnosis, Treatment, and Prevention. Philadelphia: Taylor\&Francis; 1999. p 89-101

8. Kaprio J, Koskenvuo M, Rita H. Mortality after bereavement: a prospective study of 95,647 widowed persons. Am J Public Health 1987; 77: 283-7.

9. López M, Ela M, Bartolomé N, Gómez J, García-García JA. Is the use of the health care system increased by grief?. Proceedings of the $7^{\text {th }}$ Congress of the European Association for Palliative Care; 2001 April 1-5; Palermo, Italy. Milano: EAPC; 2001. p.157.

10. García-García JA, Landa V, Trigueros MC. El proceso de duelo en atención primaria. En: De la Revilla L, ed. Libro del Año de Medicina Familiar y Comunitaria 1995. Madrid: Saned, 1995.

11. Harris T, Kendrick T. Bereavement in general practice: a survey in South Thames Health Region. Br J Gen Pract 1998; 1560-4.

12. Saunderson EM, Ridsdale L. General Practitioner' beliefs and attitudes about how to respond to death and bereavement: qualitative study. BMJ 1999; 319: 293-6.

13. Zisook, S.,\& Shuchter, S. R. (1985). The first four years of widowhood. Psychiatric Annals

14. Horowitz MJ, Weiss DS, Kaltreider N, Krupnick J, Marmar CH, Wilner N et al. Reactions to the death of a parent. Results from patients and field subjects. J Nerv Ment Dis 1997.

15. Prigerson HG, Maciejewski PK, Reynolds III ChF et al. Inventory of complicated Grief: A scale to measure maladaptative symptoms of loss. Psychiatr Research 1995;59:65-79.
16. Raphael, B. \& Martinek, N. (1993) Assessing traumatic bereavement and posttraumatic stress disorder. In J. Wilson and T. Keane, (Eds.)

17. Barreto P, Soler MC. (2007) Muerte y Duelo. Madrid. Síntesis

18. Olmeda MS. El duelo en un Centro de Salud Mental. Tesis doctoral. Universidad Autónoma de Madrid. 2000.

19. Clayton PJ, Desmarais L, Winokur G. A study of normal bereavement. Am J Psychiatry 1968;125:168-178.

20. Parkes CM. Coping with loss. Bereavement in adult life. BMJ;856-9 1998.

21. Bowlby J. La pérdida afectiva. Barcelona: Ed. Paidós; 1993.

22. Parkes CM. Bereavement. Studies of grief in adult life. $3^{\mathrm{a}}$ ed. London. \& New York: Rouledge ;1996.

23. Stroebe, W. y Stroebe, M. (1987), Bereavement and health. psychologícal and phvsical consequences of partner loss. Camhridge, Camhridge Universtty press.

24. Tizón J. Pérdida, Pena y Duelo, Editorial Pirámide. 2001, 2002, 2004.

25. Worden JW. Grief counselling and grief therapy. A handbook for mental health practitioner. London: Routledge; 1991. Traducido al castellano: Tratamiento del duelo: asesoramiento psicológico y terapia. Barcelona: Paidós 2002.

26. Camino, L., Sewell, K y Easterling, L. (2000), «Scott and Wbite grief studyphase 2: Toward an adaptive model of grief». Deatt 5/ud/es, $\mathrm{n}^{0} 24$.

27. Jacobs SC. Measures of the psychological distress of bereavement. En: Zisook S, editor. Biopsychosocial aspects of bereavement. Washington: American Psychiatric Press; 1987. p.127-38.

28. Gabriel RM, Kirschling JM. Assesing grief among bereaved elderly: a review of existing measures. Hosp J 1989; 29-54.

29. Hansson RO, Carpenter BN, Fairchild SK. Measurement issues in bereavement. En: Stroebe MS, Stroebe W, Hansson R, editores. Handbook of bereavement: Theory, research and intervention. Cambridge: Cambridge University Press; 1993. p. 62-74.

30. Faschingbauer TR, De Vaul RA, Zissok S. Development of the Texas Inventory of Grief. Am J Psychiatry 1977; 134: 696-8.

31. Faschingbauer TR. Texas Revised Inventory of Grief manual. Houston: Honeycomb Publishing; 1981.

32. García-García JA, Landa V, Trigueros MC, Calvo P, Gaminde I. El duelo por la pérdida del cónyuge: un estudio mediante grupos de discusión en atención primaria. Aten Primaria 1996.

33. García-García JA. How applicable are Anglo/American concepts of bereavement care in palliative care settings in Spain?. En: Proceedings of the $5^{\text {th }}$ Congress of the European Association for Palliative Care; 10-13 September 1997; London, U.K. London: European Association for Palliative Care; 1997.

34. García-García JA, Landa V, Trigueros MC, Gaminde I. Inventario de Experiencias en Duelo (IED): adaptación al castellano, fiabilidad y validez. Aten Primaria 2001.

35. García-García JA, Landa V. ¿Es posible medir el duelo?: adaptación al castellano y validación del Inventario de Experiencias en Duelo (IED) y del Inventario Texas Revisado del Duelo (ITRD). II Congreso Virtual de Psiquiatria, 2001.

36. STANAG 2132 (MED), sobre documentación relativa a evacuación, tratamiento y causa de muerte de pacientes (solo en Operaciones lideradas por OTAN).

37. Real Decreto 2394/2004, de 30 de diciembre, por el que se aprobaba el Protocolo para la recuperación, identificación, traslado e inhumación de los restos mortales de los fallecidos en operaciones fuera del territorio nacional.

38. Orden Ministerial 71/2010, de 15 de diciembre, por la que se crea la Unidad de apoyo a heridos y familiares de fallecidos y heridos en acto de servicio de las Fuerzas Armadas.

39. Fernández Vargas, V. (2009). El proceso de incorporación de la mujer a las Fuerzas Armadas en España. Pasado, presente y futuro. En M. Gómez e I. Sepúlveda (eds.): Las mujeres militares en España (1988-2008), 95-115. Madrid: IUGM-UNED.

40. Grabowski J, Frantz TT. Latinos and anglos: cultural experiences of grief intensity. Omega 1992-93; 26: 273-285.

\section{Otra información de interés}

Broggi, M.A., Llubià, C. y Trelis, J. (2006). Intervención médica y buena muerte. Madrid: Fundación Alternativas (www.fundacionalternativas.org)

Gracia, D. y Rodríguez Sandín, J.J., eds. (2006). Ética en cuidados paliativos. Madrid: Fundación de Ciencias de la Salud (www.fcs.es)

Hastings Center (2005). Los fines de la medicina (www.fundaciongrifols.org).

Librería virtual especializada en libros sobre la muerte y el duelo (www.alfinlibros.com ) National Consensus Project (2004). Clinical practice guidelines for quality palliative care (www.nationalconsensusproject.org). 